\title{
A New Celestial Mechanics Dynamics of Accelerated Systems
}

\author{
Gabriel Barceló \\ Dinamica Fundación, Madrid, Spain \\ Email: gestor@advanceddynamics.net
}

How to cite this paper: Barceló, G. (2019) A New Celestial Mechanics Dynamics of Accelerated Systems. Journal of Applied Mathematics and Physics, 7, 1732-1754. https://doi.org/10.4236/jamp.2019.78119

Received: July 2, 2019

Accepted: August 13, 2019

Published: August 16, 2019

Copyright $\odot 2019$ by author(s) and Scientific Research Publishing Inc. This work is licensed under the Creative Commons Attribution International License (CC BY 4.0).

http://creativecommons.org/licenses/by/4.0/

\section{(c) (i) Open Access}

\begin{abstract}
We present in this text the research carried out on the dynamic behavior of non-inertial systems, proposing new keys to better understand the mechanics of the universe. Applying the field theory to the dynamic magnitudes circumscribed to a body, our research has achieved a new conception of the coupling of these magnitudes, to better understand the behavior of solid rigid bodies, when subjected to multiple simultaneous, non-coaxial rotations. The results of the research are consistent with Einstein's theories on rotation; however, we propose a different mechanics and complementary to classical mechanics, specifically for systems accelerated by rotations. These new concepts define the Theory of Dynamic Interactions (TDI), a new dynamic model for non-inertial systems with axial symmetry, which is based on the principles of conservation of measurable quantities: the notion of quantity, total mass and total energy. This theory deduces a general equation of motion for bodies endowed with angular momentum, when they are subjected to successive non-coaxial torques.
\end{abstract}

\section{Keywords}

Mechanics, Dynamic Interactions, Non-Inertial Systems, Dynamic Coupling, Celestial Mechanics

\section{Introduction}

The observation of the dynamic equilibrium of the universe, together with the verification of the simultaneous orbitation and rotation of the celestial bodies, generated doubts about the accepted rotational mechanics.

This secular dynamic equilibrium did not seem to comply with a Newtonian physics in which the forces generate movements of constantly accelerated translation. The balance that we can observe in our universe, and its dynamics, did 
not seem to reconcile with the conceptual structure of Classical Mechanics.

With these doubts we began, from the outset of our speculations about the incoherence of the dynamic orthodox paradigm. First, we conducted a historical study on the concept of rotation in physics, published in the book: The flight of the Boomerang [1], whose prologue was written by Professor Federico Garcia-Moliner [2]. Later we published in a new book our initial concerns and hypothesis: $A$ world in rotation [3].

After observing the behavior of celestial bodies, we concluded that the application of Newtonian mechanics to mobile systems in non-inertial frames, gave conceptually wrong results.

After analyzing the physical phenomenon of a rigid solid body, free in space, subjected to multiple simultaneous non-coaxial rotations, we concluded that the current dynamics did not adequately justify the behaviors that could be observed.

In making a detailed analysis, it could not be considered that the resulting motion was chaotic; however, the true response of nature was complex and far removed from the accepted argument in the field of Classical Mechanics.

It is clear that this theory determines an ideal model for inertial systems, but is not able to justify moving systems subjected to accelerations, such as all the movements with rotation.

Therefore, it was about analyzing the existing physical-mathematical model for inertial systems and to determine a new modeling for non-inertial systems, establishing its true equation of motion [4].

The challenge was to define a new celestial mechanics based on a Dynamic of Non-Inertial Systems.

\section{Incoherences: Identification of Errors}

It is hard to believe that to date the true behavior of bodies subject to multiple, non-coaxial rotations has not been identified and that these have been assigned a mathematically unsolvable equation, even in analytical mechanics, which makes it further hard to believe that nature can behave in such a manner: without a resolvable, exact and predictive result. After our analyses, we pointed to the inconsistency in the accepted Newton-Euler equations in their application to different bodies subject to diverse non-coaxial rotations [5].

But it is also astonishing to see that the accepted calculation procedure determines a trajectory for these bodies, which does not coincide at all with what can be observed in nature (see Figure 1). This lack of coherence between the results of the formulas applied, and the true trajectory that is observed, makes us think that in all these years, no experimental tests or verifications have been carried out to confirm if those algorithms responded to reality, or if they were simple mathematical structures, alien to the authenticity of nature.

\section{Non-Inertial Systems}

Classical Mechanics has been formulated for inertial references and it does not 


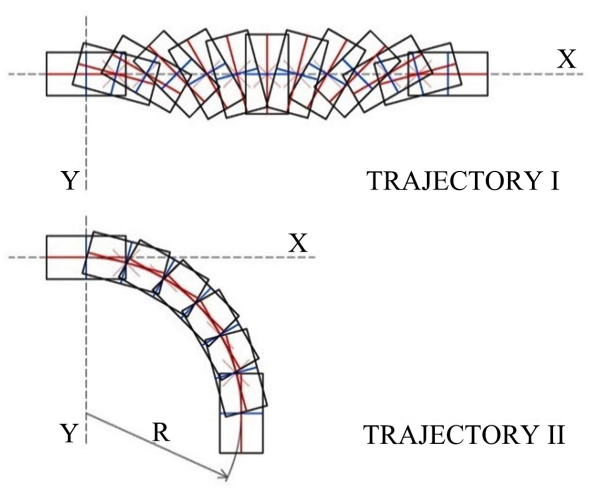

Figure 1. Trajectory I predicted by classical mechanics and trajectory II deduced by means of the Theory of Dynamic Interactions (TDI). We have observed and confirmed the second case repeatedly by experiments [6].

say anything and it does not relate to rotation cases. Nevertheless, creating a mechanics available for any stage is possible if we include their inertial reactions and we define a Fields Inertial Dynamic. In this way the inertial phenomena would be structured rationally, and incorporate a unified mechanics.

In order to include the inertial phenomena in the physics knowledge structure, it is necessary to analyze the movement in non holonomous coordinates and the axial reactions which are produced. We understand that a classical mechanics based on holonomous coordinates and polar reactions, will only represent a limited and partial natural view.

The proposed generalization does not say that the classical mechanics is outdated or wrong, but it that it is limited and partial, because it is only referred to inertial systems. We can be more aspiring and search more general dynamical laws which regulate the movement bodies behavior under rotations or, supporting multiple and non-coaxial rotations.

The Theory of Dynamic Interactions generalizes the gyroscopic couple concept and other inertial phenomena concepts and it includes into a unified structure of the new rotational non inertial dynamic [7].

\subsection{Matching Orbiting and Rotation Movements}

However, the fact is that, in general, celestial bodies in addition to orbiting also rotate about their main axis of symmetry. Nevertheless, this peculiar feature fails to have attracted the attention of astronomers until our day.

At the beginning of our project, we came to the conclusion that there could be a nomological, physical-mathematical correlation between the simultaneous rotation and orbiting movements that we observe in celestial bodies [4].

In our studies it was confirmed that it is easy to see simultaneous movements of intrinsic rotation and orbitation in nature, when until now there was no physical or mathematical model that established a scientific correlation between both movements [8].

Julio Cano has expressed: By observing in nature the constancy of the relationship 
between orbiting and intrinsic rotation, Gabriel Barceló deduced the principle that: Everything that orbits, rotates [9]; or rather, everybody that moves through an orbit simultaneously rotates on an intrinsic axis. He deduced this principle from observing the planetary system, the rings of Saturn and also the behaviour [10] of the spinning top.

He understood, however, the need for empirical checks to confirm or rectify the new dynamic hypotheses deduced from the aforementioned principle and, where appropriate, to be able to explain that behaviour by formulating a new dynamic theory that would simultaneously resolve other Rotational Dynamics phenomena and generalize inertial phenomena [6].

The Theory of Dynamic Interactions allows to justify this constant coincidence between orbiting and intrinsic rotation, and to develop a specific dynamics for bodies in rotation, subjected to successive non-coaxial torques, in which the sequence of the action of the forces is decisive, and their behavior, does not exactly coincide with the laws of Classical Mechanics.

\subsection{New Hypotheses}

Guided along by our curiosity and the intellectual concerns that our teacher Miguel A. Catalán shared with us, we have tried, over this last sixty years to seek for explanations to our doubts and aporias, trying to transform our conjectural baggage into a logical structure of knowledge to better understand the behavior of nature, to be able to accurately explain it and to come up with the mathematical tools that will make it possible to predict it [5].

To achieve this, it was necessary to observe the singular behavior of the material when subjected to successive accelerations by rotation, not coaxial, and infer their true behavior in non-inertial conditions.

From determinate dynamics assumptions and based on a new interpretation of the bodies with intrinsic angular momentum, when they are supporting successive non-coaxial torques, we have developed new dynamics hypothesis which allows us to reach the conclusion to create a new mathematical model in the rotation dynamics fields.

This new model would allow us to justify dynamic natural behaviors that until now have not been sufficiently understood. With this new conceptual model, new results for determinate cases are obtained based on a new interpretation of the composition of movements originated by external momentums [7].

Our research project was born of physical observation, the search for a dynamic for accelerated systems based on the Scientific Method, and the reflection on the validity of classical mathematical models, which accept to apply vector algebra to angular magnitudes. Therefore, from the beginning, our new differentiating hypotheses were:

- The mathematical model for the development of the classical movement equations admits the vectorial algebra, although the rotational dynamical magnitudes are neither commutative nor associative. 
- In Classical Mechanics it is accepted from Poinsot, the discriminant principle of non-overlap between the movements of translation and rotation, and that the effects of the forces do not match the effects of the torques, without this supposed rule had been experimentally tested.

\subsection{New Deductions}

Dissatisfied as we were with the criteria of conventional rotational dynamics, we tried to come up with new approaches to determine the equation of motion of accelerated systems and to better understand and explain their behavior. After a first analysis, we became convinced that:

- Whenever a solid rigid body with intrinsic rotation is subject to an external, non-coaxial momentum, dynamic interactions are generated that cause a variation in the dynamic behavior of the body, which is not provided for in classical mechanics.

- Solid bodies with intrinsic angular momentum and a quantity of movement, when they are subject to a new, non-coaxial momentum with its rotation, change their path in accordance with a defined regularity [5].

\subsection{The New Equation of Motion}

Based on the axioms put forward in this deductive process, we came to a new formulation [11] for the movement of accelerated systems: the motion equation would be determined by the translational velocity of the body's center of mass, which has not varied in magnitude and therefore will be equal to the initial translational velocity of the body subjected to the spatial rotation mentioned above:

$$
\vec{v}=\overrightarrow{\vec{\Psi}} \cdot \vec{V}_{0}
$$

The no discriminating coupling proposed in my hypothesis is hence identified as a spatial rotation of velocity:

$$
\vec{v}=\left(\begin{array}{ccc}
\cos \alpha & -\sin \alpha & 0 \\
\sin \alpha & \cos \alpha & 0 \\
0 & 0 & 1
\end{array}\right) \vec{V}_{0}
$$

Thus, the equation of motion can be written as

$$
\vec{v}=\vec{\Psi} \cdot \vec{V}_{0}=\left(\begin{array}{ccc}
\cos \left(M^{\prime}\right) t /(I \omega) & -\sin \left(M^{\prime}\right) t /(I \omega) & 0 \\
\sin \left(M^{\prime}\right) t /(I \omega) & \cos \left(M^{\prime}\right) t /(I \omega) & 0 \\
0 & 0 & 1
\end{array}\right) \vec{V}_{0}
$$

In a single rotation, the rotational operator $\vec{\Psi}$ transforms the initial velocity $\vec{V}_{0}$ into the velocity $\vec{v}$, both situated in the same plane. We find that the rotational operator $\overrightarrow{\vec{\Psi}}$ is a function of sine or cosine of $\Omega t$, which clearly indicates the relation between the angular velocity $\vec{\Omega}$ of the orbit and the torque $\vec{M}^{\prime}$ and the initial angular velocity $\vec{\omega}$. Thus, I have derived a simple mathematical relation between the angular velocity $\vec{\omega}$ of the body and its translation- 
al velocity $\vec{v}$. Equation (3) is a general equation of motion for bodies with angular momentum that are subjected to successive noncoaxial torques. For this equation, the rotational operator $\overrightarrow{\vec{\Psi}}$ serves as a matrix that transforms the initial velocity, by means of rotation, into the velocity that corresponds to each successive dynamic state [12].

This new equation of motion makes it possible to learn of the true and real behavior of nature for non-inertial systems. It can be understood as a scientific realization that explains and predicts the behavior of accelerated dynamic systems.

... This new conception of dynamics requires an interpretative sensitivity to rotational phenomena, to which the criteria of translational dynamics cannot be applied. Perhaps it corresponds to that necessary subtle change of perspective, something that we all have misse ... [13], referred to by Penrose [5].

\subsection{Dynamic Coupling}

To arrive at the different results from the physical-mathematical model obtained, we worked off the basis of a new interpretation the concept of dynamic coupling. We put forward new criteria with respect to the compounding or superposition of the movements originated by the acting forces [4].

On the basis of the Principle of Conservation of Momentum, we can infer that the field of inertial reactions generated in the rotating space by a new non-coaxial momentum, upon a moving body with a rotational movement $\omega$ and an inertial momentum I upon that rotation axis, and thus with an angular momentum, will oblige the moving body to acquire a precession rate $\Omega$.

This precession rate $\Omega$ can be observed simultaneously with the initial $\omega$, which stays constant within the body. Beyond this, and as a discriminant hypothesis, in the case of the translational movement of the body, we propose the dynamic hypothesis of the coupling of this field of translation velocities with the anisotropic field of inertial velocities caused by the second non-coaxial momentum, obtaining as a resultant movement, an orbiting that is simultaneous with the intrinsic rotation of the moving body. This new orbiting movement, generated by a non-coaxial momentum, defines itself through the rotation of the speed vector, the latter being kept constant in module.

Consequently, dynamic effects can be associated with speed, thus highlighting the clear mathematical correlation between rotation and translation. This mathematical correlation allows us to identify a physical relationship between the transfer of kinetic rotational energy to kinetic translational energy, and vice versa [4].

This is the concept of dynamic coupling used in our analysis: they are our working hypotheses. We do not understand that other possible coupling concepts should be analyzed. In this new non-inertial rotational dynamics based on the Theory of Dynamic Interactions, we have developed laws and corollaries [14] (see Treaty), which allow us an unknown number of new technological applica- 
tions.

\subsection{Brief Summary of the Theory}

In the Treaty in two volumes: New paradigm in Physics [15], we propose this new physico-mathematical model for bodies subjected to multiple actions that generate non-coaxial rotations, proposing a new structure of this knowledge.

We also state the laws that determine the behavior of systems in accelerated motion, for example, when they are subjected to non-coaxial moments, and multiple examples of assumptions and phenomena of nature that respond to these laws are proposed.

The Theory of Dynamic Interactions (TDI) explains the behavior of rotating bodies; the reason why, when these bodies are subject to new stimuli that do not share their axis of rotation, an intriguing, different and simultaneous movement is generated. This refers to a behavior that remained unexplained by the conventional laws of physics known until then [16].

Figure 2 illustrates an imaginary field of vectors that represent a supposed dynamic magnitude, for example, the speed of translation of a body, in each of its points.

It is necessary to emphasize that the fields of velocities that are the object of our analysis will be within the mobile under study, and represent that specific magnitude, in each point of the body. If the mobile is subject to a simple translation, without any rotation, the field will be uniform and isotropic, and all the vectors that represent the magnitude of that field will be equal and parallel.

On the other hand, a torque or external moment creates a new velocity field within the mobile. This field will be anisotropic, if the body already has another non-coaxial rotation, since the speed of each point will depend on its geometric position with respect to the axis, in the body that rotates.

Our dynamic hypothesis is that the velocity field of the initial rotation remains constant, but instead, the field of translation speeds is coupled with the field of translation speeds generated by the second rotation, not coaxial.

In this way, after this superposition of fields, the body changes trajectory, and if the external action remains constant, it will describe an orbit.

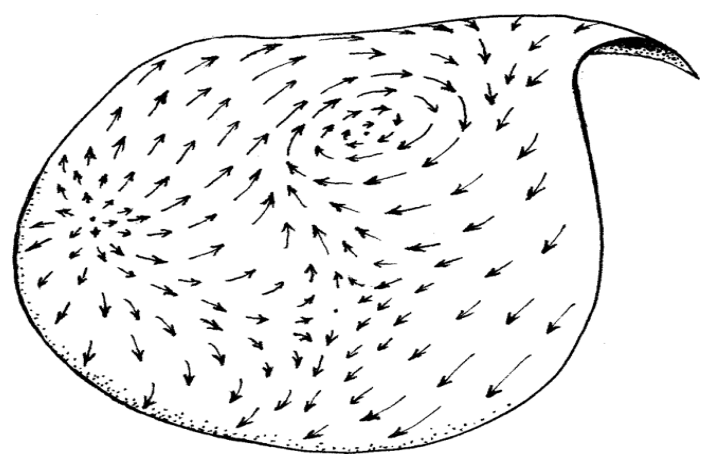

Figure 2. Example of a geometric interpretation of a field of vector dynamic magnitudes generated at the core of the mobile [4]. 
The conclusion of our proposed theory is that for this assumption, the mobile, will gradually change its trajectory, as shown in Figure 3 [17].

As we can see in Figure 3, we can assume a body with rectilinear trajectory, equipped with translational velocity and intrinsic rotation on its principal axis of inertia, which is subjected to a new non-coaxial moment with the intrinsic rotation, for example in the figure, a flotation/weight torque, contained in the drawing plane, as in our submarine experiment [18] [19] [20].

In such case, the anisotropic velocity field, generated by this flotation/weight torque, forces the mobile to rotate about a vertical axis, perpendicular to that of the external torque acting on it (See Figure 3). In red is the displaced mobile, but with the previous orientation, and in blue, the new orientation of the mobile, due to the dynamic coupling taking place. The result is the coupling of both the translational and the anisotropic fields and, consequently, the change in the mobile trajectory, describing an orbit, if its initial velocity was constant [4].

\section{Experimental Tests}

This new logical structure was contrasted with experimental tests and computer simulation models, obtaining a full coherence between the results of the simulations and the observation of the results of the empirical evidences. These tests were carried out by the Advanced Dynamics research team, but also by independent third parties [21], who designed their own experimental testing prototypes [22].

These tests can be visualized in the videos that appear in the annex I. The last video made [23], shows one of the multiple experimental examples of the Theory of Dynamic Interactions, but it is possibly one of the simplest and most striking: a cylinder operated by a finger, which acts on one of its edges. When you push the boat or cylinder with your finger, you rotate it on its longitudinal axis (main torque), and simultaneously also, on a vertical axis (secondary torque).

In the video you can see the velocity fields that are generated. There is a main angular momentum (shown with arrows), a secondary angular torque perpendicular to the previous one (shown with arrows), and a rectilinear velocity of the

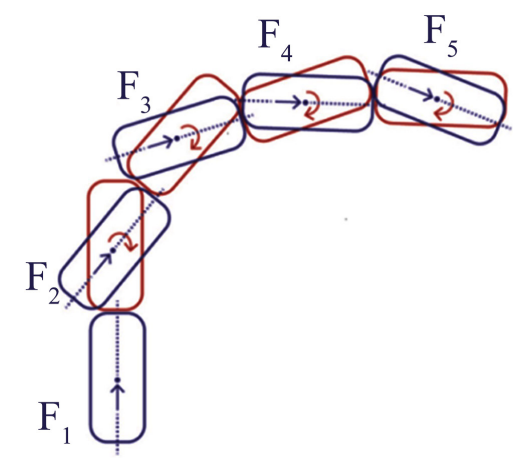

Figure 3. Trajectory of a body equipped with translational speed and intrinsic rotation on its principal axis of inertia, when it is subjected to a new non-coaxial moment with the intrinsic rotation. 
center of mass of the boat (shown with arrows). Both rotations, and the translation of the center of mass of the boat cause it to rise, without the need for an external force.

The boat rises with a tendency to become vertical; it can even stand supported on its base, in a stable position on the flat surface of the ground, but without the need for any external force acting in that direction. When the second rotation enters into action, the velocity distribution in the cylinder particles no longer remains constant, but is variable, in accordance with what is stated in the Theory of Dynamic Interactions. The generation of a variable velocity field implies the appearance of accelerations.

The velocity field generated by the second pair will have a vertical component that is coupled with the field of the speed of translation, and forces the center of mass of the cylinder to rise, that is, the movement observed is opposite to the action of the weight on the cylinder, whose tendency would be to fall. This video is an evident proof of the theory that is sustained, and we recommend its visualization: https://www.youtube.com/watch?v=hJSbVOHRfrU.

These experimental references, and many others that may arise, infer the existence of another rotational dynamics, not Newtonian, necessary for the identification of the behavior of bodies in rotation, when they are subjected to new non-coaxial stimuli, and to which their behavior. Currently, in many cases, it is understood as anomalous, paradoxical or chaotic, since the laws available to us do not allow us to identify and predetermine it [24].

\subsection{Verification and Falsifiability}

We understand that, whatever the case, our theory, as an alternative to the other classical paradigm, must be subject to independent checks and tests, owing to the clear conceptual discrepancy that exists between them. We believe it necessary to revise this niche of knowledge from a new mindset; one which discards preconceived ideas of a translational scenario and accepts the rotational reality of matter and our environment. Indeed, that has been the main reason that led us to establishing more general axioms to analyze the dynamics of bodies subject to accelerations and, especially, to acceleration by rotation, and to propose a mechanics for non-inertial systems [5].

Both the experimental tests carried out are easily reproducible according to the scientific method. Advanced Dynamics has convened three successive competitions for the possible refutation or antithesis of the proposed theory, without obtaining an answer. The last call for antithesis ended on June 15, 2019 [25].

\subsection{Justification}

Through repeated experimental tests [26], they reliably saw how certain accepted mathematical formulations do not faithfully show the true dynamic behavior of bodies subject to accelerations by simultaneous non-coaxial rotations [8].

On the contrary, the sustained theory justifies and explains many scientific concepts that could not be understood using Classical Mechanics. 
TDI fully explains the planar orbits Kepler proposed. In the laws of classical mechanics, there is no mathematical correlation which relates the movements of orbit and rotation of the planets around the Sun. However, the question of the existence of a physical relation between both movements, which has to date not been mathematically shown can be raised; ... the TDI can even explain it.

For example, TDI also explains how flat rings with multiple satellites are often formed in our solar system, such as Saturn ones ... Newton was also unable to explain the reason why Saturn's rings, and the many other ring systems in our Solar System, are flat.

Our theory can also explain the reason for its formation and even the ripples that occur in Saturn rings, and all the ring systems in general, owing to changes or disturbances in the acting momenta [4].

Also, the same reasoning could be applied to understand the behavior of many solid elements in rotation such as the boomerang [27] [28], spinning top [29], hoop, gyroscopic pendulum [30], or wheel. The Advanced Dynamics team has made multiple analyzes of these behaviors, especially in the case of the boomerang [31], having published publishing some of the results of these investigations [32].

\section{New Paradigm}

In the Treaty New paradigm in Physics, as a summary of our investigations, a new mechanical theory has been proposed for bodies subjected to accelerations; also exposing its content, and remembering how this new niche of scientific knowledge has been managed.

After this process of deduction and inference, we have built a knowledge structure for non-inertial systems that incorporates a causal demonstration of accelerated phenomena, without having knowledge to date, as we have stated, of any explicit refutation or antithesis to our reasoning. By means of a pondered management of this knowledge, based on established principles and axioms, we have structured our end thesis, which we call the Theory of Dynamic Interactions (TDI).

We believe that our theory provides a clear, satisfactory and scientific explanation of the rotational phenomena of bodies with axial symmetry [5].

Numerous articles [33] and papers have been published in congresses [34]. Also a Web on the content of the treaty: https://newparadigminphysics.com.

Different videos have been developed to describe its content:

https://www.youtube.com/watch?v=GapMJEfHJjUç;

https://www.youtube.com/watch?v=45kUpdAXICw;

https://www.youtube.com/watch?v=Gbx5wdQqTTs;

https://www.youtube.com/watch?v=Gbx5wdQqTTs.

\section{Innovations and Conclusions}

The result of our research project is the proposal of multiple conceptual and technological innovations for accelerated systems, based on the new equations, 
which we have deduced for the movement accelerated by rotations, in non-inertial dynamics.

We maintain that, in the case of systems subject to accelerations, the laws of Classical Mechanics, foreseen for inertial systems, cannot be applied, and new axioms and laws of dynamic behavior, which have been enunciated, are necessary.

\subsection{Renovating Proposals}

Below we list some of the ideas expressed in this work, that we believe could prove innovative:

\section{1) Non-discriminating coupling}

As a result of the new axioms and proposed laws, we deduce that in rotational dynamics, it is necessary to admit the non-discriminative coupling of dynamic magnitudes expressed in Section 3.4, which is represented in Figure 1, and which generates the orbital movement, simultaneous with the intrinsic rotation of the mobile.

But this dynamic, we understand that it is applicable to the cosmos, since in it, celestial bodies, in addition to having movement of translation, rotate and orbit. In addition, the rotational inertia will maintain that constant rotation, while other external actions do not prevent it: This is the reason for the secular rotation of the celestial bodies. Any new non-coaxial external action will generate orbiting movements, and not a translational expansion.

\section{2) Law of simultaneity of orbiting and rotation movements}

In our investigation we have observed and enunciated a regularity in the behavior of the celestial bodies, proposing a law of simultaneity of orbit and rotation. We reiterate that the Theory of Dynamic Interactions allows to justify that constant coincidence between orbiting and intrinsic rotation.

\section{3) Immutable dynamic balance}

One of the characteristics of our new conception of the cosmos is its constant and lasting dynamic equilibrium, because of the real behavior of matter, when bodies are endowed with intrinsic rotation.

It is the balance that the human being has perceived, when observing the celestial dome, for millennia.

\section{4) Flat celestial systems and Spiral structures}

The theory justifies the reason for the formation of rings, and in general of all the celestial plane systems that we observe in our universe.

If we look closely at the universe, we see that it is constituted by flat celestial systems. The galaxies have a flat structure, solar systems like ours, Saturn's rings, the asteroid belt, Kuiper's belt or the Scattered Disk. Precisely, the dynamic model of the TDI, justifies a flat image of the universe, in which galaxies and systems also tend to develop in flat structures [36].

The theory also justifies spiral structures, such as the arms of galaxies (See Figure 4), or tornadoes [37]. It can be understood that these structures are the expression of numerous bodies with accelerated speed and intrinsic rotation, 
subjected to non-coaxial moments.

\section{5) Oriented point}

In Classical Mechanics the abstract concept of "point" of Euclidean geometry is used, however, this concept is not ideal, and it is not enough to identify the behavior of bodies in rotational dynamics.

It is necessary to accept the use of a new concept of oriented point, to be able to integrate the inertial behavior of the matter in rotational dynamics, and to incorporate, in addition to its situation, and its orientation, the variation of this. This oriented point of the rotational dynamics is identified with 10 coordinates: $x_{1}, x_{2}, x_{3}, \varphi_{1}, \varphi_{2}, \varphi_{3}, \theta_{1}, \theta_{2}, \theta_{3}$, and time $t$, thus having 10 degrees of freedom.

\section{6) Fictitious forces and phenomenological effects}

In our research project we have repeatedly rejected the conceptual use of fictitious forces. We do not understand that in the structure of mechanical knowledge fictitious, apparent or supposed forces must be incorporated, nor that the observed behaviors can be justified with phenomenological examples, such as the gyroscopic effect.

To the current Classical Mechanics, it has been adding unstructured and phenomenological effects, which are not deduced from the initial axioms. We understand that Mechanics must constitute a structure of complete knowledge, without it being necessary to explain it with phenomenological effects, or with fictitious forces.

TDI justifies gyroscopic phenomena by field theory. The gyroscopic torque and the gyroscopic reactions are not a special and singular phenomenology of mechanics, but part of the deductive structured knowledge, of the non-inertial mechanics of accelerated systems.

\section{7) Adding angular momenta}

The increase in angular momentum may generate a new movement, different and simultaneous with the existing one, which we will call the precession movement, see Figure 5. We can remember the words expressed by Alejandro Álvarez Martínez: On observing the behavior of moving objects in rotation, when they are exposed to new non-coaxial rotations, Dr. Barceló reaches the conclusion that angular movements of non-coaxial momenta do not necessarily couple [36].

This would be the case of the top and the gyroscope, and in the case of a body in space, without ligatures such as the boomerang, the body will initiate an orbit, without the necessary existence of a central force.

\section{8) Physical-mathematical model: Inertial reactions}

In the rotational dynamics, inertial reactions within the mobile are generated, defined and regular, which have been incorporated into the logical and scientific structure of the TDI.

These reactions are incorporated by analyzing the fields of the generated dynamic magnitudes. For example, the field of translation speeds that each external action generates in the body is determined, and the real coupling of the fields that occur in nature is allowed. 


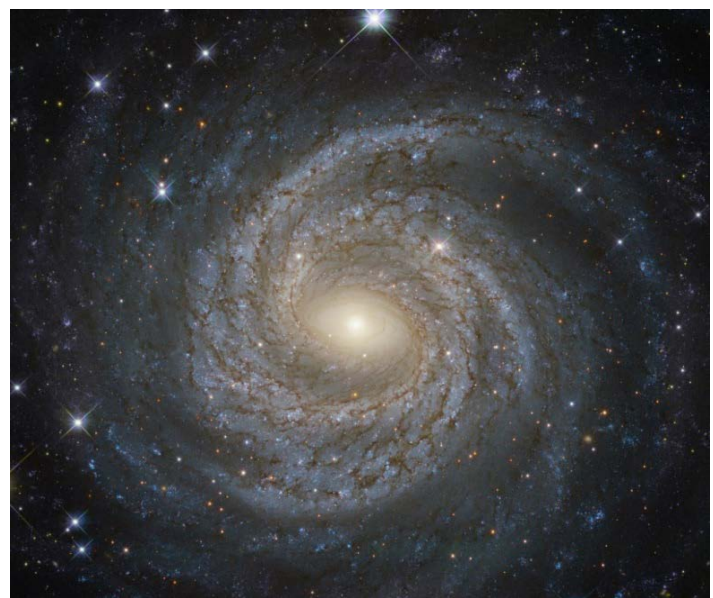

Figure 4. Spiral galaxy NGC 6814, whose arms, according to the provisions of the TDI, will be constituted by celestial bodies in rotation and accelerated translation [35].

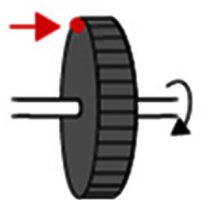

Force applied at this point

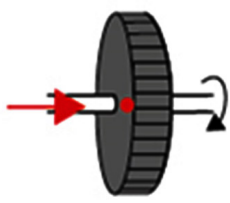

The rotor reacts as if the force had been applied at that point

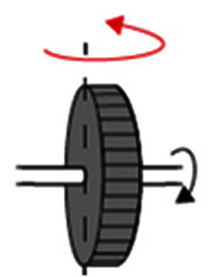

The gyroscope would make this movement

Figure 5. Precession is the response of the mobile to any external action non-coaxial with its own spin. The consequence of this reaction is that the mobile with previous intrinsic rotation modifies the result of the action, apparently displacing the point of application of the force by $90^{\circ}$, in direction of rotation of the object [17].

From these concepts, a new physical-mathematical model in rotational dynamics has been designed to understand the behavior of nature under accelerated conditions, including simultaneous non-coaxial rotations. This model is complemented with axioms, laws and corollaries, and with a new deduced movement equation.

The initial dynamic hypotheses, and the new physico-mathematical model proposed in rotational dynamics, were confirmed with the experimental tests carried out.

\section{9) Energy transfer}

The TDI confirms that it is possible to associate dynamic effects to speed and justifies a clear mathematical correlation between rotation and translation. This mathematical coupling allows us to identify a physical relationship between the kinetic rotational energy transfers to kinetic energy of translation, and vice versa.

\section{0) Contributions to the Theory of Relativity}

According to the General Theory of Relativity, we can estimate that the mass of the Earth distorts space-time in its surroundings. In this case, we can assume the analogy that the Moon makes a rolling movement on the curved surface of 
the space-time deformed by the Earth, generating a new rotation of the satellite, which we can suppose is not coaxial to the intrinsic rotation that it already has.

In this case, the dynamic interactions predicted by the TDI would be generated, resulting in the closed and flat orbit of the Moon that we see. In this way, we justify the behavior of the celestial bodies, in accordance with the criteria of relativity, without needing torques or forces.

In this same area, the second Law of Kepler can also be justified, since, in the case of an elliptical orbit, it must have a cause according to the TID, in a variation of the orbital velocity, which is consistent with the greater distortion of space-time in the vicinity of the central mass [38].

However, according to the TDI: We suggest that an observer can identify whether or not a body was previously in a state of absolute rest or absolute absence of rotation, and this leads us to the conclusion that movement does not necessarily have to be a relative concept.

This all leads us to propose that the Equivalence Principle is fully valid for the situation put forward by Albert Einstein, but cannot be generalised to all other cases of moving bodies [39].

We propose a revision of the mathematical models deduced from the General Theory of Relativity, incorporating the dynamic criteria of the TDI and its equation of the movement.

\section{1) Planck constant}

The concept of Planck radiation quantization, and its constant, understood as the smallest amount of energy that can be transmitted, could be related to the notion of spin, and be explained with the help of the TDI.

We have even proposed that this minimum value of the physical quantities could be justified by the value of the angular momentum of the atomic particles. We suggest a detailed analysis of this proposal, even applying a geometric analogy, and assuming an intrinsic rotation speed of these atomic particles, in such a way that the constant is proportional to the time necessary for a complete rotation, or even a fraction of rotation.

Therefore, TDI ... can influence the quantization concept of Planck radiation, and its constant, ... We even proposed that this minimum value of physical quantities can be justified by the angular momentum value of atomic particles [17]. This could be a new line of research based on the results obtained in this project.

\section{2) A new Celestial Mechanics}

It is thus, according to the TDI, an observable universe in which we find ourselves, with celestial bodies in constant rotation and describing orbits in space, but maintaining a dynamic equilibrium, except for exceptional situations.

This new vision of the universe, we exposed it in Imago Universi [40], and in the article: Proposal of New Criteria for Celestial Mechanics [41]. It is a new conception of Celestial Mechanics based on dynamic non-inertial hypotheses, for bodies accelerated by rotations. 


\subsection{Applications}

In previous texts, we have proposed that, through this analysis, the nature of any movement in space can be determined and predicted, defining its relativity. The movement equation that is proposed, and the laws that are formulated, permit the initiation of the structuring of a rational mechanics and of a rotational dynamics based on principles and axioms, for bodies submitted to accelerations by rotations, clearly differentiated from classical mechanics.

In this new rational structure, phenomena that are paradoxical or alien to the main structure should not be present as happens in classical mechanics with the so called gyroscopic torque or fictitious forces.

The Theory of Dynamic Interactions is a logical-deductive system constituted from some dynamic hypotheses. By means of the observation of nature, the establishing of some initial hypotheses, and starting from axioms and postulates, we have constructed a structure of knowledge in relation to rigid solid bodies, when submitted to successive accelerations by rotation.

The physical-mathematical model obtained allows us to interpret the observable behavior of these bodies, subject to successive non coaxial torques, according to deduced laws, as well as to extract new consequences, inferences and predictions. For example, the theory allows justifying the deviation that undergoes the horizontal curvilinear trajectory of a ball, when it is submitted to non-coaxial moments (See Figure 6) [38].

This text and the videos that accompany it, provide only a brief summary of the work and studies carried out to propose a Rotational Dynamics of Interactions applicable to accelerated bodies, even those subjected to multiple successive moments of force, not coaxial [42].

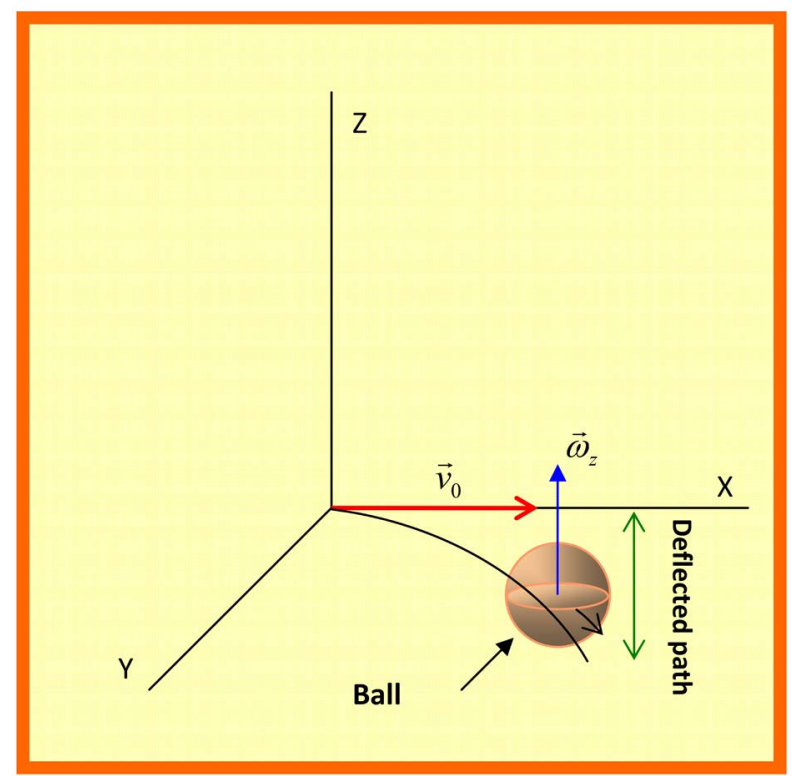

Figure 6. Horizontal curvilinear trajectory of a ball with effect, whose deviation can be justified by the Theory of Dynamic Interactions [4]. 


\subsubsection{Scientific Applications}

The Theory of Dynamic Interactions can have a transcendental effect on the foundations of dynamics [43], but also on mechanics, atmospheric dynamics, atomic physics, etc. It generalizes the concept of gyroscopic torque, and that of other inertial phenomena, incorporating them into the unified structure of a new non-inertial rotational dynamics.

Through this model of dynamic interactions, one could justify how a body in rotation can initiate an elliptical, circular or even helical trajectory, without the existence of a true central force. According to this dynamic model, the application of a torque of forces to a body with intrinsic rotation, generates a stable system, and in constant dynamic equilibrium.

The result of this project is the conception of an innovative dynamic, but also the demonstration of a rational theory of fields, which allows a new understanding of the behavior of matter [38]. The application of these dynamic hypotheses to astrophysics [44], astronautics [45] and other fields of physics and technology will possibly allow new and stimulating advances in research [46].

\subsubsection{Technological Applications}

This theory can also have numerous technological applications in the control of movement, in astronautics, in nuclear fusion plants [47] or to interpret climatological phenomena with masses of fluids in rotation, such as typhoons or tornadoes [37].

With the help of the Theory of Dynamic Interactions, a ship without a spade rudder, with energy savings, or a spaceship could be governed [48]. This theory can also be applied in the confinement of plasma, in nuclear fusion reactors [49].

Also has numerous and significant ... applications, especially in orbital dynamics, orbit determination, and orbit control. For instance:

- Variation of the affecting torque, arises when subjecting intrinsic angular momentum bodies to new non-coaxial momentums.

- To conceive an intrinsic rotating mobile solid, which could be exclusively controlled due to Dynamic Interactions.

- To calculate the trajectory of any intrinsic angular momentum solid in space.

- To propose a new steering system independent from a rudder or any other external element [38].

We can suggest advances in the studies and application related to orbital mechanics, guidance, navigation, and control of single or multi-spacecraft systems as well as space robotics and rockets [24].

\subsection{Conclusions}

It is necessary to admit the existence of a rotational dynamics of interactions with real results and which modifies the behavior of bodies in accordance with some specific and universal dynamic Laws.

We want to suggest that interest should arise in physics in the exploration of non-inertial accelerated systems, and also to express a call for the need to devel- 
op scientific investigation projects for their evaluation and analysis, as well as technological projects based on these hypotheses. In our opinion, these hypotheses suggest new keys to understand the dynamics of our environment and the harmony of the universe. A universe composed not only of forces, but also of their momentums; and when these act constantly upon rigid rotating bodies, with an also constant translation speed, the result is a closed orbiting movement, thus a system which is moving, but within a dynamic equilibrium.

The application of these dynamic hypotheses to astrophysics, astronautics and to other fields of physics and technology possibly allows new and stimulating advances in investigation.

The result of this project is the conception of an innovative dynamic theory, which specifically applies to rigid rotating physical systems and which has numerous and significant scientific and technological applications.

The Theory of Dynamic Interactions establishes new conceptual criteria, of a more general description, to understand the behavior of nature, meaning that the current laws of dynamics could be considered special and specific cases of this theory. For example, Newton's laws would apply to the case of a physical model of behavior, without force momentums [38].

We have also referred to examples in nature, which support the Theory of Dynamic Interactions, supporting the proposed laws [14].

Next, we will list, a series of characteristics of the physical theory that is proposed. It can be understood that this relationship is a proposal of conclusions, without pretending to be exhaustive:

\section{1) Criteria}

The initial hypotheses of the theory are based on new interpretative criteria, for example, on speed coupling and rotational inertia, and on a new mathematical model, which allows the simulation of the real behavior of the bodies subjected to these excitations.

As we have already expressed, our hypotheses have been confirmed by experimental tests.

\section{2) Content}

The Theory of Dynamic Interactions is a logical-deductive ordering of knowledge, based on certain dynamic hypotheses. Through the observation of nature, the establishment of initial hypotheses, and the statement of principles, axioms and postulates, we have constructed a structure of knowledge in relation to rigid solid bodies, when subjected to successive accelerations by rotation.

The mathematical physical model obtained allows us to interpret the observable behavior of these bodies, subjected to successive non-coaxial pairs, according to deduced laws, as well as to extract new consequences, inferences and predictions.

\section{3) Harmony of the Universe}

We have tried to imagine the trajectory of a body in rotation in space, when it is forced to make new rotations on new axes, obtaining as a result the orbital iti- 
nerary that we observe in the celestial bodies, and a balance and secular harmony of our universe. A universe that is not necessarily expanding, according to the TDI [43].

\section{4) Field theory}

The result of this research project is the conception of an innovative dynamic, and the exposition of a rational field theory, which provides a new understanding of the behavior of matter.

Our research can be expanded, in addition to the study of Field Theory, with a deep relativistic analysis, which allows knowledge of the dynamics of new physical systems of space, and the potential future achievement of numerous and relevant new technological developments.

\section{5) Consistency with classical mechanics}

This theory does not intend to challenge Newton's laws; since with them a conceptual structure of proven reliability has been developed. We propose a different and complementary mechanics to Classical Mechanics, specifically for systems accelerated by rotations. Therefore, both theories do not come into conflict, as they do not refer to the same domain of knowledge. They are theories of specialization, in their respective fields of action.

We propose a new Celestial Mechanics based on a specific rotational algebra for non-inertial areas, in which the initial hypotheses of the laws of the translational movement of Classical Mechanics are not respected. We propose the exploration of a new knowledge niche for specific dynamic conditions, but not trivial, because they can be observed repeatedly in our universe.

It is necessary to admit the existence of a rotational dynamics of interactions, with real results, and that modifies the behavior of bodies according to universal dynamic laws that were not known to date.

\section{6) Correlation}

In our research project, a total coherence can be found between the initial speculations, the original hypotheses, the principles and axioms applied, the physical laws deduced, even causal laws that justify the observed behavior, the mathematical physical models corresponding to the equations of movement which result from the dynamic laws deduced, the simulation models achieved, and the experimental tests carried out. There are videos with these experimental tests (See Appendix).

We believe that with this new model that we propose, it will facilitate the understanding of our observational universe, and of the physical phenomena that we notice in it.

We want to suggest the interest that the exploration of accelerated non-inertial systems should have in physics, and express a call to the need to develop scientific research projects in this field, for their evaluation and analysis, as well as technological projects based on these hypotheses.

To obtain more information about this theory, we suggest going to the books and texts referred to and also visiting the following portals: 
https://newparadigminphysics.com/

http://www.advanceddynamics.net/

http://www.dinamicafundacion.com/

http://www.tendencias21.net/fisica/

https://club.tendencias21.net/mundo/

http://imagouniversi.com/

\section{Conflicts of Interest}

The author declares no conflicts of interest regarding the publication of this paper.

\section{References}

[1] Barceló, G. (2006) The Flight of the Boomerang. Ed. Marcombo, Barcelona.

[2] Garcia-Moliner, F. (2017) Physical-Mathematical Models in Rotational Motions. World Journal of Mechanics, 7, 35-38. https://doi.org/10.4236/wjm.2017.73004 http://www.scirp.org/Journal/PaperInformation.aspx?PaperID=74661

[3] Barceló, G. (2008) A Rotating World. Editorial Marcombo, Barcelona. http://www.dinamicafundacion.com

[4] Barceló, G. (2018) New Paradigm in Physics, Volume II: Assumptions and Applications of the Theory of Dynamics Interactions.

[5] Barceló, G. (2017) New Paradigm in Physics, Volume I: Theory of Dynamics Interactions. Agencia del ISBN, Madrid.

[6] Cano, J. (2015) The Pendulum of Dynamic Interactions. Journal of Applied Mathematics and Physics, 3, 1186-1198. http://www.scirp.org/journal/jamp https://doi.org/10.4236/jamp.2015.39146

[7] (2016) Research Blog: Extensive Report on the Investigations of Gabriel Barceló. Global Journal of Researches in Engineering. http://blog.gjre.org/2016/03/behaviour-of-rotational-bodies.html

[8] Barceló, V. (2017) A Scientific Legacy: Theory of Dynamics Interactions. World Journal of Mechanics, 7, 85-100. https://doi.org/10.4236/wjm.2017.73009 http://www.scirp.org/Journal/Home.aspx?IssueID=9235\#74661

[9] Barceló, G. (2006) The Flight of the Boomerang. Ed. Marcombo, Barcelona, 311. http://www.dinamicafundacion.com

[10] http://www.advanceddynamics.net/spinning-top-video

[11] Barceló, G. and Álvarez, A. (2007) Mathematical Model in Rotational Dynamics. International Congress of Mathematics in Engineering and Architecture. Polytechnic University of Madrid, T. S. Civil Engineering, Madrid. http://advanceddynamics.net/congresos-reuniones-y-conferencias

[12] Barceló, G. (2012) Analysis of Dynamic Fields in Non-Inertial Systems. World Journal of Mechanics, 2, 175-180. https://doi.org/10.4236/wjm.2012.23021

[13] Penrose, R.R. (2006) The Road to Reality: A Complete Guide to the Laws of the Universe. Débate, Barcelona.

[14] Barceló, G. (2013) Theory of Dynamic Interactions: Laws of Motion. World Journal of Mechanics, 3, 328-338. https://doi.org/10.4236/wjm.2013.39036

[15] https://www.amazon.com/dp/846979518X/ref=rdr_ext_tmb

[16] Dalby, F. (2017) Rolling Over into the Age of Algorithm. World Journal of Me- 
chanics, 7, 39-42. https://doi.org/10.4236/wjm.2017.73005

http://www.scirp.org/Journal/PaperInformation.aspx?PaperID=74663

[17] Barceló, G. (2018) Theory of Dynamic Interactions. Global Summit on Physics Congress, Madrid, 27 September 2018, 1-31.

https://dinamicafundacion.com/wp-content/uploads/2018/10/GSP2018ENG.pdf

[18] https://www.youtube.com/watch?v=dtMqGSU9gV4\&feature=related

[19] https://www.youtube.com/watch? $\mathrm{v}=\mathrm{qK} 5 \mathrm{~mW} 2 \mathrm{j} 2 \mathrm{nzU} \&$ feature=related

[20] https://www.youtube.com/watch?v=vSUkd4slHGQ

[21] https://vimeo.com/68763196

[22] Pérez, L.A. (2013) New Evidence on Rotational Dynamics. World Journal of Mechanics, 3, 174-177. http://www.scirp.org/journal/wjm https://doi.org/10.4236/wjm.2013.33016

[23] Pérez, L.A. (2018) Cylinder Subjected to Two Non Coaxic Rotations. https://www.youtube.com/watch?v=hJSbVOHRfrU

[24] Barceló, G. (2010) On the Equivalence Principle. In: 61 st International Astronautical Congress, American Institute of Aeronautics and Astronautics, Prague.

[25] http://www.coiim.es/forocientifico/FORO\%20CIENTFICO/Documentos/ON_THE _EQUIVALENCE_PRINCIPLE.pdf

[26] https://www.youtube.com/watch?v=P9hGgoL5ZGk\&feature=related

[27] Barceló, G. (2014) Theory of Dynamic Interactions: The Flight of the Boomerang. Journal of Applied Mathematics and Physics, 2, 569-580.

https://doi.org/10.4236/jamp.2014.27063

http://www.scirp.org/journal/jamp

[28] Barceló, G. (2015) Theory of Dynamic Interactions: The Flight of the Boomerang II. Journal of Applied Mathematics and Physics, 3, 545-555.

https://doi.org/10.4236/jamp.2015.35067

http://www.scirp.org/journal/jamp

[29] Alejandro, Á.M. and Almudena, M.G. (2016) The Dance of the Spinning Top. Global Journal of Science Frontier Research: A Physics \& Space Science, 16, 15-27.

https://globaljournals.org/GJSFR_Volume16/E-Journal_GJSFR_(A)_Vol_16_Issue 3.pdf

[30] https://www.dropbox.com/s/rrjb1786ub75a8h/PIDing_m.mp4?dl=0 http://www.advanceddynamics.net/the-pendulum-video

[31] https://vimeo.com/129383447

[32] Almudena, M.G. (2017) The Flight of the Boomerang: Comments. World Journal of Mechanics, 7, 46-67.

http://www.scirp.org/Journal/Home.aspx?IssueID=9235\#74661

[33] Merino, J. (2017) The Works and Days of Gabriel Barceló. World Journal of Mechanics, 7, 43-45. https://doi.org/10.4236/wjm.2017.73006 http://www.scirp.org/Journal/PaperInformation.aspx?PaperID=74664

[34] Barceló, G. (2009) Dinámica de sistemas acelerados por rotación XXXII Reunión Bienal de la R.S.E. de Física. Ciudad Real, septiembre.

http://www.advanceddynamics.net

[35] ESA/Hubble \& NASA (2017) Acknowledgement: Judy Schmidt. https://www.nasa.gov/image-feature/goddard/2016/hubble-spies-a-spiral-snowflake

[36] Alejandro, A.M. (2017) Theory of Dynamic Interactions: Innovations. World Journal of Mechanics, 7, 101-119. https://doi.org/10.4236/wjm.2017.73010 
[37] Barceló, G. (2014) Dynamic Interactions in the Atmosphere. Atmospheric and Climate Sciences, 4, 828-840. https://doi.org/10.4236/acs.2014.45073 http://www.scirp.org/Journal/PaperInformation.aspx?PaperID=51584\#.VHB4YTSG To

[38] Barceló, G. (2017) Theory of Dynamic Interactions: Synthesis. Transactions on Machine Learning and Artificial Intelligence, 5, 10-25. http://scholarpublishing.org/index.php/TMLAI/article/view/3344

[39] Barceló, G. (2014) On Motion, Its Relativity and the Equivalence Principle. Journal of Modern Physics, 5, 1839-1847. https://doi.org/10.4236/jmp.2014.517180 http://www.scirp.org/Journal/PaperInformation.aspx?PaperID=51422\#.VHB0jzSG_ To

[40] Barceló, G. (2013) Imago Universi. Arpegio, Barcelona. https://dinamicafundacion.com/imago-universi/?lang=en

[41] Barceló, G. (2013) Proposal of New Criteria for Celestial Mechanics. International Journal of Astronomy and Astrophysics, 3, 385-391. https://doi.org/10.4236/ijaa.2013.34044

[42] Cano, J. (2017) Rotational Dynamics: A Challenge. World Journal of Mechanics, 7, 68-84. http://www.scirp.org/Journal/Home.aspx?IssueID=9235\#74661

[43] The Universe Does Not Necessarily Expand Forever. https://www.alphagalileo.org/en-gb/Item-Display/ItemId/161784?returnurl=https:// www.alphagalileo.org/en-gb/Item-Display/ItemId/161784

[44] Barceló, G. (2006) A New Rotational Dynamics of Interactions for the Planet Saturn.

http://dinamicafundacion.com/wp-content/uploads/2014/02/UNA-NUEVA-DINA MICA-ROTACIONAL-DE-INTERACCIONES-PARA-EL-PLANETA-SATURNO. pdf

[45] Barceló, G. (2005) Dynamic Anomalies in the Pioneer Space Probes. RSEF's 2005 XXX Biennale.

http://dinamicafundacion.com/wp-content/uploads/2014/02/ANOMAL\%C3\%8DA S-DIN\%C3\%81MICAS-EN-LAS-SONDAS-PIONEER1.pdf

[46] Barceló, G. (2011) Analysis of Dynamics Field Systems Accelerated by Rotation. Dynamics of Non-Inertial Systems. DeMSET-2011 Congress, Miami, 30 November 2011.

http://www.coiim.es/forocientifico/FORO\%20CIENTFICO/Documentos/DeMSET 2011_GBarcelo.pdf

[47] Barceló, G. (2014) Dynamic Interaction Confinement. World Journal of Nuclear Science and Technology, 4, 249-260. https://doi.org/10.4236/wjnst.2014.44031 http://www.scirp.org/journal/PaperInformation.aspx?paperID $=51026 \&$

[48] Barceló, G. (2013) Technological Applications of the New Theory of Dynamic Interactions. Global Journal of Researches in Engineering. Mechanical and Mechanics Engineering- $G$, 13, 33-39.

https://globaljournals.org/GJRE_Volume13/E-Journal_GJRE_(G)_Vol_13_Issue_5. pdf

[49] Barceló, G. (2016) Dynamic Interaction: A New Concept of Confinement. Global Journal of Science Frontier Research: A Physics \& Space Science, 16, 1-9. https://globaljournals.org/GJSFR_Volume16/E-Journal_GJSFR_(A)_Vol_16_Issue_ 3.pdf 


\section{Appendix}

\section{Experimental Tests and Videos}

Several test experiments have been conducted over recent years, the results of which have been fully satisfactory. These tests serve to confirm the dynamic hypotheses that underpin the Theory of Dynamic Interactions. Videos have been recorded of these tests that can be seen at the following links:

Barceló, G.: Theory of Dynamic Interactions. Videos, 2002.

http://www.youtube.com/watch?v=P9hGgoL5ZGk\&list=PL3E50CF6AEBEED $\underline{47 \mathrm{~B}}$

http://www.youtube.com/watch?v=XzTrGEtJGXU\&list=PL3E50CF6AEBEED $\underline{47 \mathrm{~B}}$

http://www.youtube.com/watch?v=dtMqGSU9gV4\&list=PL3E50CF6AEBEED $\underline{47 \mathrm{~B}}$

http://www.youtube.com/watch?v=qK5mW2j2nzU\&list=PL3E50CF6AEBEED47 $\underline{B}$

Bauluz, E.: New Dynamic Hypotheses. Madrid, 2011. This video showed the experiments carried out by Advanced Dynamics S. A. to prove and justify the http://www.youtube.com/watch?v=vSUkd4slHGQ

Sanchez Boyer, J.: Imago Universi. Video, Madrid, 2013.

https://vimeo.com/62247544

Pérez, L. A.: Reflecting New Evidence on Rotational Dynamics, 2013. Video. http://vimeo.com/68763196

Sanchez Boyer, J.: The Flight of the Boomerang II, Video. 2015.

https://www.youtube.com/watch?v=mGfrGW5fhOg\&feature=youtu.be https://vimeo.com/129383447

Pérez, L. A.: The Pendulum of Dynamic Interactions. Video. 2015. https://vimeo.com/160873005

Pérez, L. A.: The Dance of the Spinning Top. Video, Valladolid, 2015. www.advanceddynamics.net/spinning-top-video/

Pérez, L. A.: Cylinder Subjected to Two Non Coaxic Rotations. 2018. https://www.dropbox.com/s/wgb6oztjvdcnziy/EBP_EN.mp4?dl=0

Web Advanced Dynamics: Animations and Videos

http://advanceddynamics.net/en/medios-audiovisuales/ 3.0 Submarine Simulation.

3.1 Submarine Prototype I.

3.2 Submarine Anisotropic Field.

3.3 Resultant Field.

3.4 Prototype II.

3.5 Prototype II Simulation.

3.6 Prototype Prototipo II navigation.

3.7 Catamaran.

4.0 Translation Velocity Field.

4.1 Generating a New Field. 
4.2 Resultant Field.

4.3 Velocity Coupling.

4.4 End Velocity Field.

$5.01^{\text {st }}$ Simulation.

$5.12^{\text {nd }}$ Simulation.

The Pendulum of Dynamic Interactions

Theory of Dynamic Interactions: The Flight of the Boomerang II Dynamic Interaction Theory Presentation Videos (4)

New Dynamic Hypotheses

New Evidential Proof of Rotational Dynamics 